\title{
Development of a Micropropagation Protocol for St. John's Wort (Hypericum perforatum L. )
}

\author{
Joe-Ann McCoy and N.D. Camper ${ }^{1}$ \\ Department of Plant Pathologyand Physiology, Clemson University, Clemson, \\ SC 29634-0377
}

Additional index words. tissue culture, medicinal plant

\begin{abstract}
Hypericum perforatum L. (St. John's Wort) has an extensive history as an important medicinal herb used for the treatment of neurological and depressive disorders (Linde et al., 1996). The objective of this study was to establish an in vitro tissue culture protocol for St. John's Wort. Nodal segments, axillary buds, and leaf disc explants produced multiple shoots and callus on Murashige and Skoog minimal organics medium supplemented with combinations of indoleacetic acid (IAA; 0.57, 2.85, 5.71 $\mu \mathrm{M}$ ) and benzylaminopurine $(\mathrm{BA} ; 2.22,4.44,8.88 \mu \mathrm{M})$. Shoot production occurred on all combinations of IAA/BA tested and was significantly less in treatments without hormones. Callus production was higher on treatments containing $2.85 \mu \mathrm{M} \mathrm{IAA}+4.44 \mu \mathrm{M} \mathrm{BA}$, or 5.71 $\mu \mathrm{M} \mathrm{IAA}$ $+8.88 \mu \mathrm{M}$ BA. Shoots transferred to hormone-free medium at 8 weeks formed roots by 12 weeks. A micropropagation protocol was established for St. John's Wort using mature plants as the explant source.
\end{abstract}

St. John's Wort products are currently among the top five selling phytopharmaceuticals in North America (Blumenthal, 1999). St. John's Wort has historically been harvested for its upper flowering portions that are used to treat neurological and mild depressive disorders (Linde et al., 1996). The majority of plant material supplied to phytopharmaceutical companies has traditionally come from native populations in the Pacific Northwest or from foreign markets. Advantages to growing St. John's Wort commercially may include higher yields per hectare, more efficient harvesting, and stable chemical composition through selective breeding. Unfortunately, cultivars that are bred for increased secondary product accumulation are not necessarily disease resistant. Micropropagation of St. John's Wort could provide a rapid multiplication method for producing plants exhibiting desirable characteristics. These characteristics include disease resistance, increased secondary product accumulation, and beneficial morphology. In field situations, St. John's Wort blooms and is harvested the second year of cultivation; field plants are replanted every 2 to 3 years.

St. John's Wort has been successfully micropropagated (Cellarova et al., 1995; Murch et al., 2000; Zdunek and Alfermann, 1992) utilizing aseptically grown seedlings as an explant source. The objectives of the study reported herein were to: 1) develop a micropropagation protocol for St. John's Wort utilizing organized tissue from mature plants

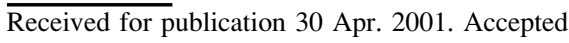
for publication 7 May 2002. Technical contribution No. 4623 of the South Carolina Agricultural Experiment Station. Supported in part by USDA NRI Grant 96-35501-3297.

${ }^{1}$ Corresponding author; Dept. of Plant Pathology and Physiology, Clemson Univ., Clemson, SC 29634-0377. and 2) evaluate shoot, root, and callus production.

\section{Materials and Methods}

Parental stock St. John's Wort plants (20, 4-month-old) were purchased from Richters Nursery Co. (Goodward, Ontario, Apr. 1997). Ten plants were transferred to $15-\mathrm{cm}$ pots and placed in a growth chamber under controlled growing conditions $\left(22-28 \mu \mathrm{mol} \cdot \mathrm{m}^{-2} \cdot \mathrm{s}^{-1}\right.$ at 24 $\left.{ }^{\circ} \mathrm{C}\right)$. The remaining 10 plants were planted in an irrigated field. Growth-chamber-grown plants were used as the explant source for all experiments described herein.

Three types of explants were used. 1) Nodal segments: stem sections including nodes (3-4 $\mathrm{cm})$ were washed in antibacterial soap for 30 min followed by a sterile distilled water rinse for $4 \mathrm{~h}$. Segments were surface-sterilized by immersion in a $10 \%$ aqueous solution of $5.4 \%$ sodium hypochlorite with Tween-20 (three drops $/ 100 \mathrm{~mL}$ ) for $8 \mathrm{~min}$ followed by three rinses in sterile distilled water, placed in sterile petri plates and excised into $1.5-\mathrm{cm}$ sections with damaged ends and axillary shoots removed. Segments (one per tube) were vertically implanted onto medium containing one of four hormonal combinations in $95 \times 25-\mathrm{mm}$ glass culture tubes containing $10 \mathrm{~mL}$ medium. 2) Axillary buds: stem sections $(1-3 \mathrm{~cm})$ of mature growth-chamber plants were washed with antibacterial soap and distilled water for $10 \mathrm{~min}$ followed by a sterile distilled water rinse for $3 \mathrm{~h}$. Stem sections were then surfacesterilized by immersion in a $5 \%$ aqueous solution of $5.4 \%$ sodium hypochlorite with Tween20 (Fischer-Biotech, Fischer Scientific, N.J.) for $5 \mathrm{~min}$ followed by three rinses in sterile distilled water. Axillary buds were then excised from the stem axil and aseptically placed into culture tubes (one per tube) containing 10 $\mathrm{mL}$ of one of four hormonal combinations. 3 )
Leaf discs: leaf discs were excised from young leaves of growth-chamber plants and washed in sterile water with antibacterial soap for 10 min followed by a 1-h immersion in sterile distilled water with constant agitation. Leaves were surface-sterilized by immersion in a $5 \%$ aqueous solution of $5.4 \%$ sodium hypochlorite with Tween- 20 for 4 min followed by three rinses in sterile distilled water. Leaf discs were excised with a 6-mm cork borer and transferred onto sterile disposable petri plates containing $20 \mathrm{~mL}$ medium with one of four hormonal combinations. Discs were cultured five per plate and incubated in a growth chamber.

All cultures were incubated at $24^{\circ} \mathrm{C}$ with a $16-\mathrm{h}$ photoperiod $\left(22-28 \mu \mathrm{mol} \cdot \mathrm{m}^{-2} \cdot \mathrm{s}^{-1}\right.$ Murashige and Skoog minimal organic medium (MSMO; Murashige and Skoog, 1962) was supplemented with $25 \mathrm{~g} \cdot \mathrm{L}^{-1}$ sucrose, 1.0 $\mathrm{g} \cdot \mathrm{L}^{-1}$ casamino acid, $2.0 \mathrm{mg} \cdot \mathrm{L}^{-1}$ glycine, 6.0 $\mathrm{g} \cdot \mathrm{L}^{-1}$ agar, and one of the following four hormone combinations: control, no hormones; treatment 1: $0.57 \mu \mathrm{M}$ IAA and $2.22 \mu \mathrm{M} \mathrm{BA}$; treatment 2: $2.85 \mu \mathrm{M}$ IAA and $4.44 \mu \mathrm{M} \mathrm{BA}$; treatment 3: 5.71 $\mu \mathrm{M}$ IAA and $8.88 \mu \mathrm{M}$ BA. Medium $\mathrm{pH}$ was adjusted to 5.0 and the media autoclaved at $121{ }^{\circ} \mathrm{C}$ for $30 \mathrm{~min}$. Tests used either $96 \times 25$ shell vials or petri plates with 20 replications per explant. Experiments utilizing nodal segments and axillary shoots as explant sources were conducted three times; experiments utilizing leaf discs were conducted twice).

Shoot and callus presence and/or absence was rated weekly for a 6 -week period. Analysis of variance was used to determine the overall effect of treatment, explant, and treatment and explant interactions. Means of treatments and explants were compared using a pair-wise $t$ test $(\alpha=0.05)$ to determine significant differences among treatment and explant effects. All analyses were performed using SAS (SAS Institute, 1996).

\section{Results and Discussion}

Multiple shoot and callus formation was observed on all three explants tested and on all hormone combinations tested (Fig. 1). Analysis of variance indicated a treatment effect on callus and shoot production, but no explant effect and no explant $\times$ treatment interactions (Table 1). Shoot and callus formation were low $(0 \%$ to $15 \%)$ on the control treatment (Fig. 1). There were no significant differences between explant type. Green callus production was significantly higher on treatments 2 and 3 than on treatment 1 or the control (Fig. 1B). Regenerated plantlets produced flowers (Fig. 2B) and characteristic red-pigmented glands. These hypericin-containing glands accumulated on leaf margins, petals, and stamens of regenerated plants.

According to Stafford and Warren (1991) when meristem stability is studied, the amount of variation produced from callus derived plantlets is much greater than that found in axillary shoots proliferating from stem nodes. Propagation involving a callus stage produces higher rates of somoclonal variation and high proportions of polyploids (Stafford and Warren, 


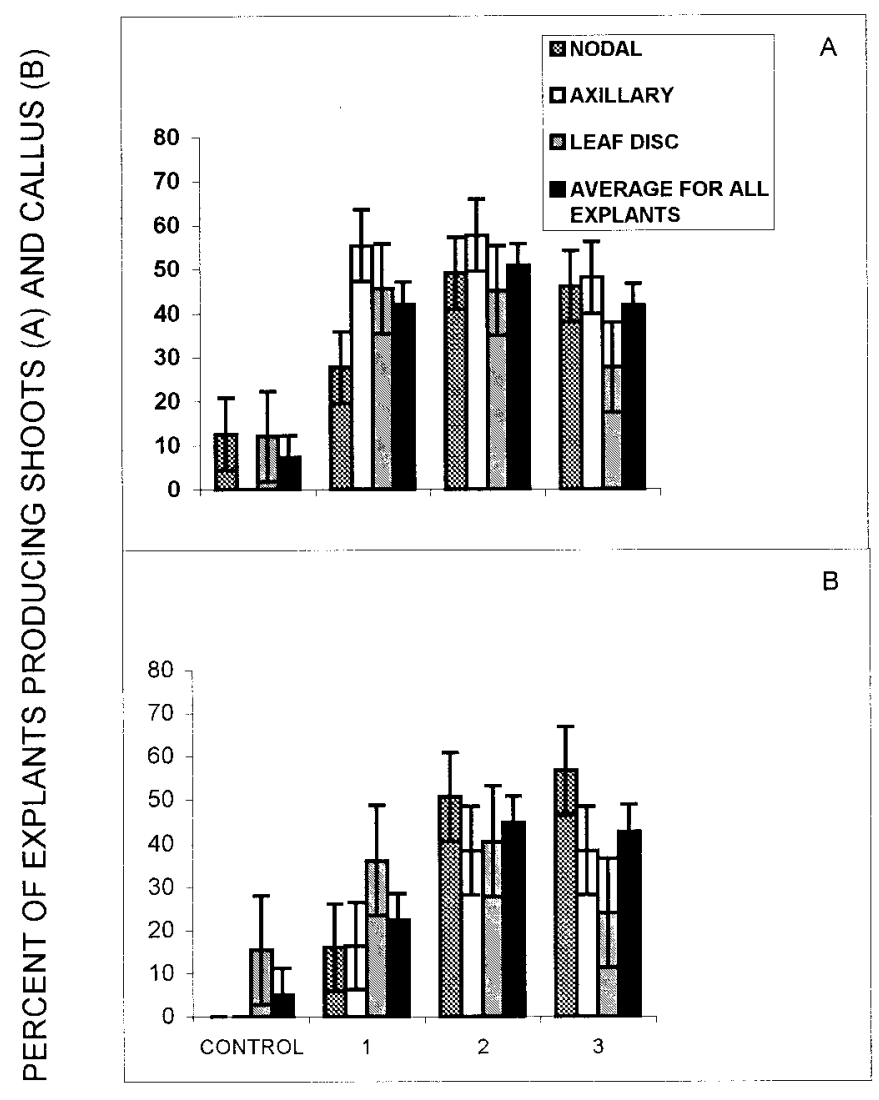

TREATMENT

Fig. 1. (A) Shoot and (B) callus formation from nodal segments, axillary buds and leaf discs of St. John's Wort as affected by different hormone combinations. Treatments consisted of: Control, no hormones; Treatment 1: $0.57 \mu \mathrm{M}$ IAA and $2.22 \mu \mathrm{M} \mathrm{BA}$; Treatment 2: $2.85 \mu \mathrm{M}$ IAA and $4.44 \mu \mathrm{M}$ BA; Treatment 3: $5.71 \mu \mathrm{M}$ IAA and $8.88 \mu \mathrm{M}$ BA. Error bars represent $\pm 1 \mathrm{SD}$.

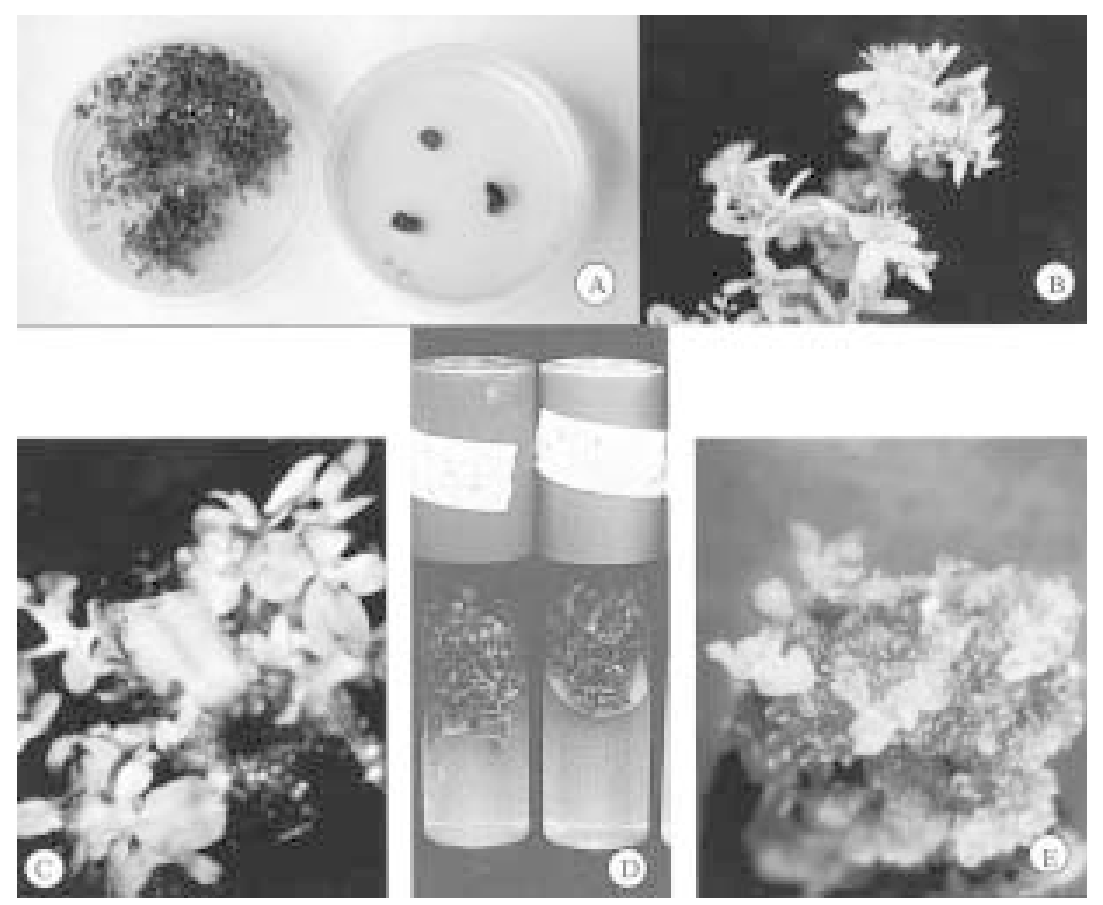

Fig. 2. Morphological stages of tissue-cultured plants of St. John's Wort: (A) leaf discs on Treatment 3: 1.0 $\mathrm{mg} \cdot \mathrm{L}^{-1} \mathrm{IAA}+2.0 \mathrm{mg} \cdot \mathrm{L}^{-1} \mathrm{BA}$, at 2 (right) and 6 (left) weeks; (B) mature in vitro regenerated plant with flowers; (C) multiple shoot formation from nodal segments on MSMO medium with 5.71 $\mu \mathrm{M} \mathrm{IAA}+4.44$ $\mu \mathrm{M}$ BA at 5 weeks; (D) multiple shoot formation on MSMO medium with $5.71 \mu \mathrm{M} \mathrm{IAA}+4.44 \mu \mathrm{M}$ BA from a 5-week-old axillary bud explant; (E) callus and shoot production on MSMO medium with $2.85 \mu \mathrm{M}$ IAA $+4.44 \mu \mathrm{M} \mathrm{BA}$, from a 4-week-old leaf disc.
Table 1. Analysis of variance for treatment and explant effects on shoot and callus production by St. John's Wort cultured on MSMO medium with different hormone combinations for 6 weeks.

\begin{tabular}{lrcc}
\hline & & \multicolumn{2}{c}{ Mean square values } \\
\cline { 3 - 4 } Variable & df & Shoot & Callus \\
\hline Replicate & 2 & $205^{\mathrm{Ns}}$ & $520^{\mathrm{Ns}}$ \\
Treatment (Trt) & 3 & $2706^{* * * *}$ & $2351^{* * * *}$ \\
Explant (Expl) & 2 & $178^{\mathrm{Ns}}$ & $181^{\mathrm{Ns}}$ \\
Trt $\times$ Expl & 6 & $313^{\mathrm{Ns}}$ & $361^{\mathrm{Ns}}$ \\
Error & 18 & 199 & 308 \\
\hline Ns, *****N Nonsignificant or significant at $P<0.0001$.
\end{tabular}

1991). Propagation from axillary shoots tends to be stable and exhibits low levels of variation. The goal of this study was to develop a protocol to propagate desirable individuals; therefore, the callus stage should be avoided. Conversely, if the objective is to generate variability in a crop, a short callus step prior to adventitious shoot induction may prove effective.

When Cellarova et al. (1995) propagated $H$. perforatum from aseptically germinated seedlings, the concentration of the cytokinin (BA) was the determining factor for regeneration capacity. Studies reported herein showed that shoot production was not significantly different in treatments containing 2.22, 4.44, or $8.88 \mu \mathrm{M}$ BA although it was significantly different from the control (Fig. 1A). Multiple shoot formation occurred between 2.22 to $8.88 \mu \mathrm{m} \mathrm{BA}$ (see Fig. 2C-D). Callus (Fig. 2E) production was higher in treatments containing 2.22 to $8.88 \mu \mathrm{M} \mathrm{BA}$ (Fig. 1B).

Time course evaluation of shoot production showed that nodal segments and axillary buds can be transferred within 3 weeks for rooting purposes (Fig. 3) whereas leaf discs should remain in culture for at least 5-6 weeks (Fig. 3). Shoots were transferred to hormonefree medium at 8 weeks with subsequent root development by week 12 (data not shown). When transferred to medium with either IAA or IBA, no roots were produced. In preliminary experiments, shoots formed roots within 1 week when transferred from shoot induction medium directly to soil and placed in a growth chamber, and showed a $70 \%$ survival rate (McCoy, 1998). This direct soil transfer would save $\approx 3$ weeks in obtaining rooted plants when compared to the hormone-free medium transfer. In this study, 4-month-old St. John's Wort stock plants that were maintained in a growth chamber produced flowers within 3 months (in June after planting in April) and bloomed throughout the winter. In contrast, 4-monthold stock plants maintained in the field produced only sporadic blooms during the first season and did not bloom consistently until the second season when they are traditionally harvested. A successful micropropagation technique established using explants from mature plants can provide a constant supply of desirable plants, which could be propagated yearround and harvested in their first year when grown under controlled conditions. Because St. John's Wort is a hemicryptophyte, its perrenating bud is at soil level (Cellarova et al., 1995); this characteristic enables it to thrive 


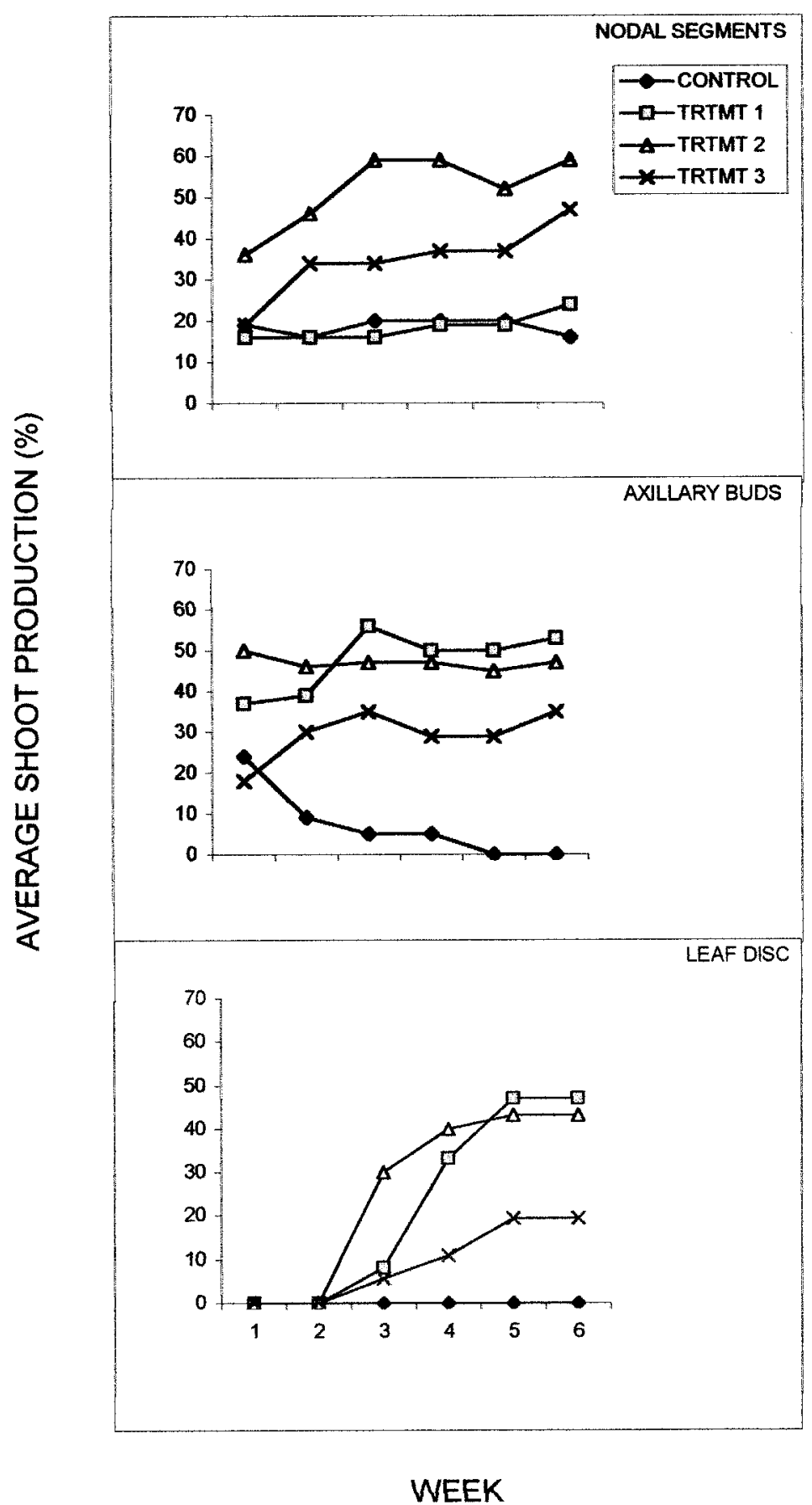

Fig. 3. Shoot production from three explants (nodal segments, axillary buds and leaf discs) of St. John's Wort as a function of time (weeks). Treatments were: Control, no hormones; Treatment 1,0.57 $\mu \mathrm{M}$ IAA and 2.22 $\mu \mathrm{M}$ BA; Treatment 2, $2.85 \mu \mathrm{M}$ IAA and $4.44 \mu \mathrm{M} \mathrm{BA}$; and Treatment 3, 5.71 $\mu \mathrm{m}$ IAA and $8.88 \mu \mathrm{M}$ BA.

from regular harvesting, which acts as pruning to benefit the plant's growth form and increased flower production. St. John's Wort could potentially be grown under greenhouse conditions and harvested throughout the year, unlike field-grown crops, which cannot be harvested until the second year and have a limited bloom season. Hypericin and pseudohypericin are the marker compounds used in establishing the quality of St. John's Wort in commercial products (Wagner and Bladt, 1994). Hypericin and pseudohypericin analysis of first-year growth-chamber plants and mature field plants showed no significant difference in content (McCoy, 1998). Analysis of callus cultures for hypericin content showed no appreciable amounts (McCoy, 1998).

For further study, it would prove useful to determine if in vitro-cultured plants share the same genetic characteristics (e.g., hypericin content) as their parent plants. Cellarova et al. (1995) found that in vitro-regenerated plants from hypocotyls and cotyledons showed greater variation when compared to controls. It would be useful to determine if plants regenerated from more organized meristematic regions, such as nodal segments and axillary buds, display similar amounts of variation, or if it is indeed reduced as suggested by Stafford and Warren (1991).

In conclusion, an effective protocol was established for the micropropagation of $H$. perforatum utilizing mature plants as the explant source. The protocol used an MSMO medium with IAA and BA for shoot production. The procedure can be used for rapid multiplication and genetic improvement of this commercially important medicinal plant.

\section{Literature Cited}

Blumenthal, M. 1999. Market report. Herbalgram 47:64-65.

Cellarova, E., K. Kimakova, Z. Daxnerova, and P. Martonfi. 1995. Hypericum perforatum (St. John's Wort): In vitro culture and the production of hypercin and other secondary metabolites, $\mathrm{p}$. 261-275. In: Y. Bajaj (ed.). Biotechnology in agriculture and forestry. Medicinal and aromatic plants III. Springer-Verlag, Berlin.

Linde, K., G. Ramirez, C. Mulrow, A. Pauls, W. Weidenhammer, and D. Melchart. 1996. St. John's Wort for depression-An overview and meta-analysis of randomized clinical trials. Brit. Med. J. 313:253-258.

McCoy, J. 1998. In vitro culture of Hypericum perforatum and analysis of hypericins. MS Thesis. Dept. of Plant Pathology and Physiology, Clemson Univ., Clemson, S.C.

Murashige, T. and F. Skoog. 1962. A revised medium for rapid growth and bioassays with tobacco tissue cultures. Physiol. Plant. 15:473497.

Murch, S., K. Choffe, J. Victor, T. Slimmon, S. KrishnaRaj, and P. Saxena. 2000. Thidiazuroninduced plant regeneration from hypocotyl cultures of St. John's Wort (Hypericum perforatum cv. Anthos). Plant Cell Rpt. 19:576-581.

SAS Institute. 1996. SAS guide for personal computers, version 6.12. SAS Inst., Cary, N.C.

Stafford, A. and G. Warren. 1991. Plant cell and tissue culture. Wiley, New York.

Wagner, H. and S. Bladt. 1994. Pharmaceutical quality of hypericum extracts. J. Geriatric Psych. Neurol. Suppl. Vol. 7. 1:S65-S68.

Zdunek, K. and A. Alfermann. 1992. Initiation of shoot organ cultures of Hypericum perforatum and formation of hypercin derivatives. Planta Medica 58:A621-A622. 